\title{
ANTIMICROBIAL SUSCEPTIBILITY; PREVALENCE OF BACTERIA ISOLATED FROM ICU PATIENTS OF TERTIARY CARE HOSPITAL. \\ drmshahbaz@yahoo.com
}

1. M.Phil (Microbiology) Assistant Professor Microbiology Sheikh Zayed Medical College/ Hospital, Rahim Yar Khan, Pakistan 2. FCPS (Medicine)

Associate Professor Medicine Sheikh Zayed Medical College/ Hospital, Rahim Yar Khan, Pakistan 3. M.Phil Pharmacology

Assistant Professor Pharmacology Sheikh Zayed Medical College/ Hospital, Rahim Yar Khan, Pakistan

Correspondence Address: Dr. Muhammad Shahbaz Hussain Assistant Professor Microbiology Sheikh Zayed Medical College/ Hospital

Rahim Yar Khan, Pakistan drmshahbaz@yahoo.com

Article received on: 26/07/2016

Accepted for publication: 15/10/2016

Received after proof reading: 00/00/2016

\section{Dr. Muhammad Shahbaz Hussain', Dr. Zafar Majeed², Dr. Mazhar Hussain ${ }^{3}$}

ABSTRACT: Infections occurring more than 48 hours after admission in hospital are usually considered as nosocomial infections. Patients in ICUs are at a higher risk of acquiring this infection than those in non-critical areas. The total length of stay increased from three days to nineteen days for clinical procedures. Increased antimicrobial resistance in such cases makes increase in mortality and morbidity. The ICU infection rates is five to ten times higher than HAls in ward patients ICU acquired infections are major health problem globally, in developing countries like Pakistan. The microorganisms that are frequently isolated from intensive care units are Acinetobacter spp, P. aeruginosa, Klebsiella pneumoniae, Streptococcus spp, Staphylococcus spp, Enterococci and Enterobacteriaceae i.e E. coli, Proteus mirabilis and Serratia marcescens. Aims: The present study was aimed at to determine the frequency of causative organisms in intensive care unit (ICU) patients and antimicrobial susceptibility pattern of the isolates. Study Design: A cross-sectional study. Place and Duration of Study: Microbiology section of Pathology department Sheikh Zayed Medical College/Hospital, Rahim Yar Khan. Four months (September to December 2015). Materials and Methods: Clinical samples from patients having signs and syptoms of site-specific infections or fever in ICU were collected. The samples were cultured on suitable culture media and bacterial isolates were identified by Gram stain and standard biochemical methods. Antimicrobial susceptibility testing to conventional and newer antibiotics was performed on Mueller Hinton agar using disc diffusion method. Frequency percentages of all isolates were determined. Results: Out of 100 samples only 30 samples were positive for growth and 70 samples showed no growth. The most frequent isolates were E. coli (40\%), K. pneumoniae (10\%) P. aeruginosa (10\%) Proteus spp. (20\%) Acinetobacter $(17 \%)$ and S.aureus (3\%). S.aureus (Gram positive bacteria) were more sensitive to imipenem, cefoxitin and resistant to ceftriaxone, nitrofurantoin azithromycin, Tetracycline, Cefotaxime and amoxicillin clavulanic acid. Other Gram negative bacteria i.e E.coli, K. Pneumoniae, P.aeruginosa, Proteus spp. and Acinetobacter spp. were sensitive to imipenem, sulzone, ceftriaxone, Ciprofloxacin, Gentamicin, Nitrofurantoin and resistant to amoxicillin clavulanic acid. Conclusion: Bacteria were isolated from $30 \%$ clinical samples taken from patients admitted in ICU. The most frequent site of infection was the respiratory tract followed by urinary tract and blood stream infections. E. coli, P. aeruginosa, K. pneumonia, Proteus spp., Acinetibacter and S. aureus were isolated. The high frequency of Health associated infections and elevated bacterial resistance rate in ICU patients suggest that more strict measures regarding infection control practices can be done.

Key words: Intensive care unit, Disc diffusion method, Antimicrobial susceptibility, Mueller Hinton Agar

Article Citation: Hussain MS, Majeed Z, Hussain M. Antimicrobial susceptibility; prevalence of bacteria isolated from ICU patients of tertiary care hospital. Professional Med J 2016;23(12):1581-1586. DOI: 10.17957/TPMJ/16.3546

\section{INTRODUCTION}

Patients in ICUs are at a high risk of getting hospital acquired infections (HAls) than those in non-critical care area. ${ }^{1}$ The increased duration of stay varies from three days for gynecological procedures to nineteen days for procedures in orthopedic wards. ${ }^{2}$
Nosocomial infections may be exogenous, the source of being any part of hospital ecosystem, including objects, people, water, food and air in the hospital. These infections are opportunistic and bacteria of low virulence can cause the disease in hospital patients who are immunocompromised. Consequently, increase in antimicrobial resistance in these cases lead to increase morbidity and 
mortality. Hospital acquired infections are caused by bacteria, viruses, fungi and parasites. ${ }^{3}$

The microbes can be moved from patient to patient (cross infection). In endogenous infection they be able to part of patient's own normal flora. In environmental transfer microbes can be transferred from an inanimate object contaminated material recentaly by the human source. ${ }^{4}$ The World Health organization (WHO) proved that the nosocomial infection rate will continue to rise as a result of 4 factors, which include hospital crowded conditions, increase number of people suffering from compromised immune system, emergence of new microbe and increased bacterial resistance. ${ }^{5}$

Acquired infection rates in intensive care units is 5-10 times higher than nosocomial infections in ward patients ICU acquired infections are main health distress globally, especially in developing countries like Pakistan. ${ }^{6}$ Rate of ICU acquired infections vary from 18 to $54 \%$ and is responsible for 5 to $35 \%$ of all Nis and about $90 \%$ of all caese of disease outbreaks in intensive care units. ${ }^{7}$

Patients who are admitted to ICUs are at an elevated risk of infection associated with urinary catheter, infections related to bloodstream and (VAP) ventilator-associated pneumonia in patients admitted to intensive care unit. ${ }^{8}$ They are considered a chief risk to patient safety and main cause of increased cost, mortality and morbidity rates. ${ }^{9}$ The most common health acquired infections in ICU patients are (UTIs) urinary tract infections, (RTIs) respiratory tract infections and (BSIs) bloodstream infections readily due to use of invasive devices. Infection rates are also higher in patients with increased susceptibility due to younger or old age, underlying disease, immunodeficiency or chemotherapy. ${ }^{10}$

The microbes that usually contribute in intensive unit care infections include Streptococcus spp., Pseudomonas aeruginosa, Acinetobacter spp., S. aureus and coagulasenegative staphylococcus, Enterococci and Enterobacteriaceae including Proteus mirablis,
E. coli, Serratia marcescens and Klebsiella pneumonia. Out of these, P. aeruginosa, E. coli, $S$. aureus and Enterococci have a chief role. ${ }^{11} \mathrm{HAls}$ are seen worldwide but are less studied and less emphasized in developing countries like Pakistan. Study conducted in Rawalpindi showed that most recurrent site of infection in ICUs was RTIs (47.95\%) followed by UTIs (25.3\%). Pseudomonas aeruginosa, Escherichia coli and Klebsiella pneumonia were the most commonest bacteria. The isolation rate of Gram-positive bacteria was relatively low. ${ }^{12}$

Antimicrobial resistance (AMR) is one of the major problems throughout the world. It is the ability of bacteria and other microbes to survive drug. The major mechanisms of AMR are mutations, target alterations, enzymes and efflux pump. Multidrug resistance (MDR) is the ability of microorganisms to resist two or more drug.

\section{MATERIALS AND METHODS}

The study was carried out in the Microbiology section of pathology department Sheikh Zayed Medical College/Hospital, Rahim yar khan, Pakistan from September to December 2015. A total of 100 samples including blood, pus, urine, catheter tips and tracheal secretions were collected from intensive care unit (ICU). Urine was inoculated on CLED agar while other samples were cultured on blood, Chocolate and MacConkey's agar plates. Plates were incubated aerobically for 18-24 hours. Primarily bacteria were identified based on colonial characteristics, Gram stain and biochemical tests (catalase test, coagulase, cytochrome oxidase and motility test).After confirmation of bacteria. The antimicrobial susceptibility testing of all isolates was done by the standard disk diffusion method using commercial disks (Oxoid) of Imipenem (10ug), amoxicillin clavulanic acid (20ug:10ug), ciprofloxacin (5ug), gentamicin (10ug), tetracyline (30ug), azithromycin (15ug) Pipracillin Tazobactam and ceftriaxone (30ug). The swab was then used to distribute the bacteria evenly over the entire surface of Mueller Hinton Agar (Oxoid). Inoculated plates were kept for a while. With the help of sterile forecep antibiotic discs 
were placed on the surface of Mueller Hinton agar. After 18-24 hours incubated plates were examined. Zones of inhibition by Vernier calipers and results were interpreted in accordance with guidelines of CLSI 2015.

\section{RESULTS}

A total of 100 samples were collected from patients admitted to ICU. Samples cosists of urine (40), pus (12), blood (25), tracheal secretion (13) and catheter tips (10) as shown in figure-1. The samples were inoculated on blood, CLED and MacConkey agar at $37^{\circ} \mathrm{C}$ incubation. After 18-24 hours, plates were examined for microbial growth. Out of these samples growth were shown by urine $37 \%$, blood $23 \%$, pus $13 \%$, catheter tips $10 \%$ tracheal secretion $17 \%$ and 70 samples shown no growth, as shown in table-1. Percentage of microorganisms isolated from samples were E.coli (40\%), K. Pneumonia (10\%) P. aeruginosa (10\%) Proteus spp. (20\%) Acinetobacter (17\%) and $\mathrm{S}$. aureus (3\%) as shown in table-1.

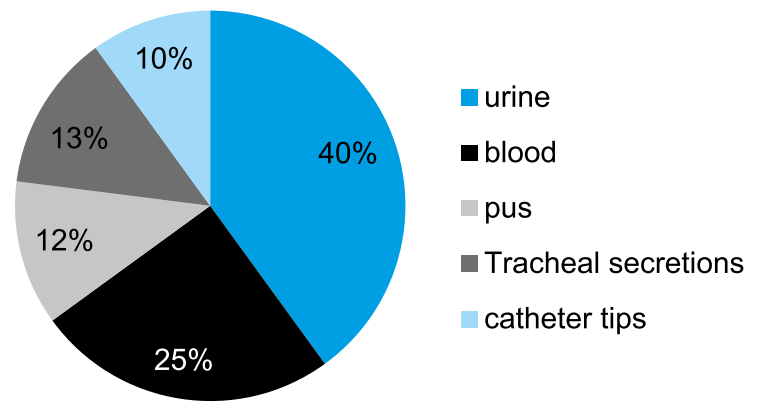

Figure-1. Percentage of sample collected from ICU patients:
Antimicrobial sensitivity pattern was performed on both Gram positive and gram negative bacteria. Gram positive bacteria such as S. aureus were highly sensitive to imipenem, Cefoxitin and resistant to ceftrixone, azithromicin and amoxicillin clavulanic acid, tetracycline nitrofurantoin and gentamicin, ciprofloxacin as shown in Figure-2.

Gram negative bacteria such as E.coli, K. Pneumoniae, P.aeruginosa, Proteus spp. and Acinetobacter were sensitive to imipenem, sulzone, ceftriaxone, Ciprofloxacin, Cefotaxime, nitrofurantoin, gentamicin, pipracillin Tazobactam, fosfomycin and resistant to amoxicillin clavulanic acid as shown in Figure-3.

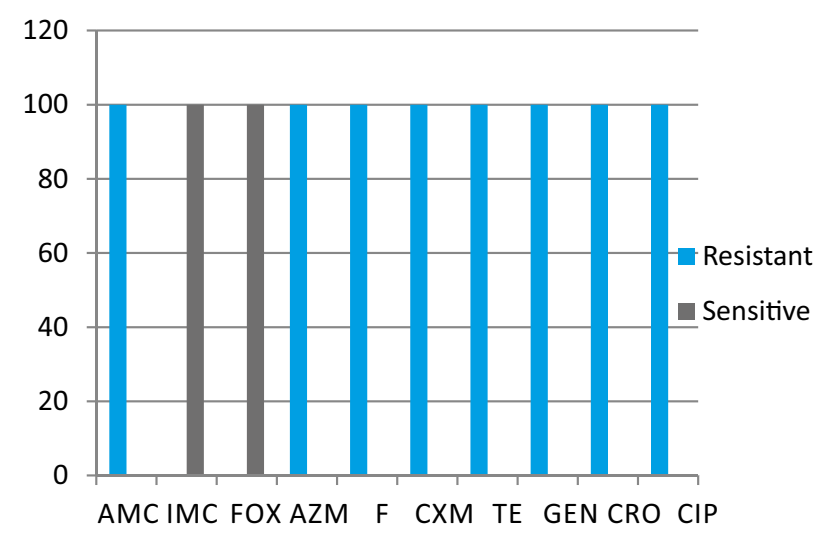

Figure-2. Antimicrobial susceptibility pattern of $\mathrm{S}$. aureus:

\section{DISCUSSION}

Infections acquired in hospitals and among health care workers, but appear after discharge are called nosocomial infections.

\begin{tabular}{|l|c|c|c|c|c|c|c|}
\hline \multirow{2}{*}{ Organisms } & \multicolumn{7}{|c|}{ Samples } \\
\hline & Blood & Urine & Pus & Catheter tips & Tracheal secretion & Total & Percentage (\%) \\
\hline E.coli & - & 8 & 1 & 1 & 2 & 12 & 40 \\
\hline K. Pneumoniae & - & 1 & 2 & - & - & 3 & 10 \\
\hline P.aeruginosa & 2 & - & - & - & 1 & 3 & 10 \\
\hline Proteus spp. & - & 1 & 1 & 2 & 2 & 6 & 20 \\
\hline Acinetobacter & 5 & - & - & - & - & 5 & 17 \\
\hline S. aureus & - & 1 & - & - & - & 1 & 3 \\
\hline Total & 7 & 11 & 4 & 3 & 5 & 30 & 100 \\
\hline
\end{tabular}




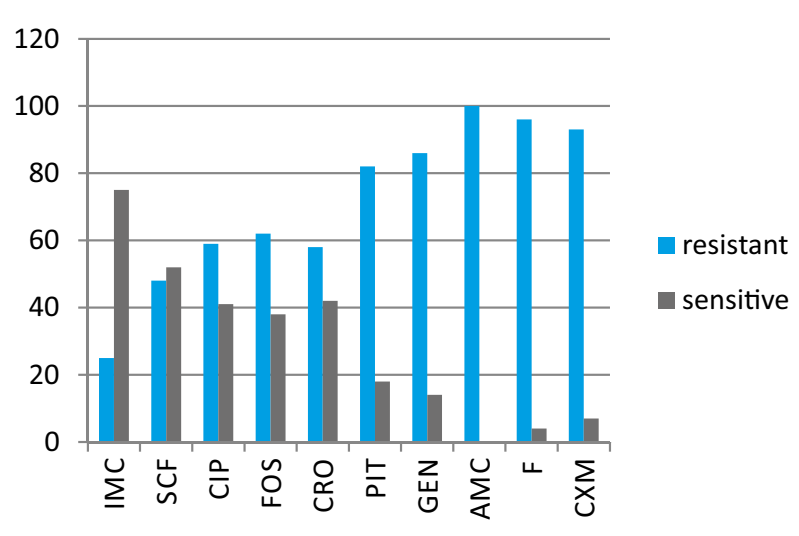

Figure-3. Antimicrobial susceptibility of E.coli, K. Pneumoniae, P. aeruginosa, Proteus spp. and Acinetobacter.

Patients in ICUs are at a higher risk of NIs due to various reasons such as trauma, multiple illness, invasive medical procedures and immunosuppression, concerning endotracheal tubes and disruption of barriers during insertion of venous catheter, branula, urinary catheter, bedsores and wound management, environmental factors for example crowding, inanimate and animate reservoirs of infection.

Out of 100 samples 30 samples were positive for growth and 70 showed no growth. The samples were urine, blood, pus, catheter tips and tracheal secretion. The most common organisms isolated were E.coli, K. Pneumoniae, P. aeruginosa, Proteus spp and Acinetobacter. Gram-positive bacteria were infrequently isolated.

NIs are seen worldwide but are less studied and are less emphasized in developing countries like Pakistan. This study reports high rate of infections, in addition to high antibiotic resistance in ICU settings of a tertiary care hospital in Rahim Yar Khan. RTIs (38.46\%) and UTIs (27.5\%) were the most common infections from these patients and this is in agreement with other studies from Pakistan. ${ }^{13}$ Rizvi et al., reported the frequency of RTIs and UTI was $21 \%$ and $44.6 \%$ respectively. The frequency of RTIs and UTIs was $30.1 \%$ and $39.1 \%$ from hyderabad. According to a study carried at Rawalpindi the frequency of RTIs and UTIs was $47.95 \%$ and $25.3 \%$ respectively. Difference should be because of the difference in antibiotic prescribing practices and variations in sample size, collection time, culture techniques and susceptibility testing. ${ }^{14}$ Relatively high rate of blood stream infections (32\%) in this study as compared to other studies $23.7 \%$ and $27 \%$ may partly because blood cultures were processed manually and blood stream infections are less prevalent in our setting. ${ }^{15}$ United States National NIs surveillance system showed that nosocomial pneumonia accounted for $31 \%$ of all $\mathrm{NIs}$ followed by UTIs and BSIs. ${ }^{16}$

Most commonly organisms isolated were $\mathrm{E}$. coli (40\%) K. Pneumoniae (10\%) P. aeruginosa (10\%) Proteus spp. (20\%) and Acinetobacter (17\%) S.aureus (3\%) in our study.A study conducted in Indonesia showed the most frequent bacteria isolated were P. aeruginosa (50.9\%) and $E$. coli (8.7\%). ${ }^{17}$ A study in tertiary care center of Saudi Arabia reported that the most frequent bacteria are Acinetobacter baumannii, P. aeruginosa, E. coli, K. pnemoniae. ${ }^{18}$ Similar studies were conducted in hospitals and several ICUs in Asian countries including Philippine, India Iran' China and Nepal demonstrated that the most frequent bacteria derived from ICU samples were P. aeruginosa, Klebsiella spp. and Staphylococcus aureus in accordance to our results. ${ }^{19}$

Gram positive bacteria showed high sensitivity to imipenem, Cefotaxime and very high resistance to the most commonly used drug sulzone, ceftrixone, gentamicin, nitrofurantoin, azithromycin and amoxicillin clavulanic acid .This may be due to misuse, overuse of these antibiotics. Imipenem, Ceftriaxone, Fosfomycin, Ciprofloxacin and sulzone were highly sensitive against Gram negative microorganisms. Comparatively lower resistance rate to imipenem Ceftrixone, Fosfomycin, Ciprofloxacin and sulzone is probably due to lesser use of these antibiotics and is in agreement with another local study. ${ }^{20}$ The high rates of antimicrobial resistance identified in the present study might be due to the lack of antibiotic use guidelines and policies

\section{CONCLUSION AND FUTURE WORK}

The most frequent site of infection from patients 
attending ICUs were RTIs followed by urinary tract and BSIs. E. coli, P. aeruginosa, K. pneumonia, Proteus spp., Acinetibacter and S. aureus were isolated. The high frequency of health acquired infections and increased resistance in ICU patients recommend that more appropriate measures regarding infection control practices should be done.

Copyright(c) 15 Oct, 2016.

\section{REFERENCES}

1. Richards M, Thursky K, Buising K. Epidemiology, prevalence, and sites of infections in intensive care units. Semin Respir Crit Care Med 2003; 24:3-22.

2. Gordis, Leon, "Epidemiology, Third Edition," Elsevier Saunders 2004.

3. Patwardhan RB, Dhakephalkar PK, Niphadkar KB, Chopade BA. A study on nosocomial pathogens in ICU with special reference to multiresistant Acinetobacter baumannii harbouring multiple plasmids. Indian J Med Res. 2008; 128:178-87. [PubMed].

4. F. Vandenesch, G. Lina, T. Henry Staphylococcus aureus hemolysins, bi-component leukocidins, and cytolytic peptides: a redundant arsenal of membrane-damaging virulence factors? Front Cell Infect Microbiol, 2 (2012), p. 12View Record in Scopus.

5. Weinstein RA. Nosocomial infection update. Emerg Infect Dis 1998; 4:416-20.

6. Rosenthal VD, Guzman S, Orellano PW. Nosocomial infections in medical-surgical intensive care units in Argentina: attributable mortality and length of stay.

7. Festary, V. Kourí, C.B. Correa, D. Verdasquera, T. Roig, M.P. Couret cytomegalovirus and herpes simplex infections in mothers and newborns in a Havana maternity hospital MEDICC Rev, 17 (1) (2015), pp. 29-34.

8. Digiovine B, Chenoweth C, Watts C, Higgins M. The attributable mortality and costs of primary nosocomial bloodstream infections in the intensive care unit. Am J Respir Crit Care Med 1999 Sep; 160 (3):976-981.

9. Mesiano ER, Merchán-Hamann E. Bloodstream infections among patients using central venous catheters in intensive care units. Rev Lat Am Enfermagem 2007 May-Jun; 15 (3):453-459.

10. Aly NY, Al-Mousa HH, Al Asar SM. Nosocomial infections in a medical-surgical intensive care unit. Med Princ Pract 2008; 17 (5):373-377.

11. T.C. Horan, M. Andrus, M.A. Dudeck CDC/NHSN surveillance definition of health care-associated infection and criteria for specific types of infections in the acute care setting Am J Infect Control, 36 (5) (2008), pp. 309-332.

12. Dr. Naeem Akhtar, Hospital Acquired Infections in a Medical Intensive Care Unit in Department of Pathology, Rawalpindi, Medical College, Rawalpindi Journal of the College of Physicians and Surgeons Pakistan 2010, Vol. 20 (6): 386-39.

13. Rizvi MF, Hasan $Y$, Memon AR, Abdullah M, Rizvi MF, Salee $\mathrm{m} \mathrm{S}$, etal. Pattern of nosocomial infection in two intensive care units of a tertiary care hospital in Karachi. J Coll Physicians Surg Pak 2007; 17:136-9. Comment in: J Coll Physicians Surg Pak 2008; 18:1345.

14. Richards M, Thursky K, Buising K. Epidemiology, prevalence and sites of infections in intensive care units. Semin Respir Crit Care Med 2003; 24:3-22.

15. Shaikh JM, Devrajani BR, Shah SZ, Akhund T, Ishrat $B$. Frequency, pattern and etiology of nosocomial infections in intensive care unit: an experience at a tertiary care hospital. J Ayub Med Coll Abbottabad 2008; 20:37-40.

16. Richards MJ, Edwards JR, Culver DH, Gaynes RP. Nosocomial infections in combined medical-surgical intensive care units in the United States. Infect Control Hosp Epidemiol. 2000; 21: 510-5.

17. Winarto Prevalence of extended-spectrum -lactamases (ESBL)-bacteria of blood isolates in Dr. Kariadi Hospital Semarang 2004-2005. Media Medika Indosiana.2009; 43(5):260-267.

18. Al Johani SM, Akhter J, Balkhy $\mathrm{H}$, El-Saed $\mathrm{A}$, Younan M, Memish Z. Prevalence of antimicrobial resistance among gram-negative isolates in an adult intensive care unit at a tertiary care center in Saudi Arabia. Ann Saudi Med. 2010; 30:364-369. [PMC free article] [PubMed].

19. Litzow JM, Gill CJ, Mantaring JB, Fox MP, MacLeod WB, Mendoza $\mathrm{M}$, et al. et al. High frequency of multidrugresistant gram-negative rods in 2 neonatal intensive care units in the Philippines. Infect Control Hosp Epidemiol. 2009; 30(6):543-549.

20. Wahid F, Masood N, Jafri A. Nosocomial pneumonia in mechanically ventilated patients. Pak Armed Forces Med J 2005; 3:25-8. 


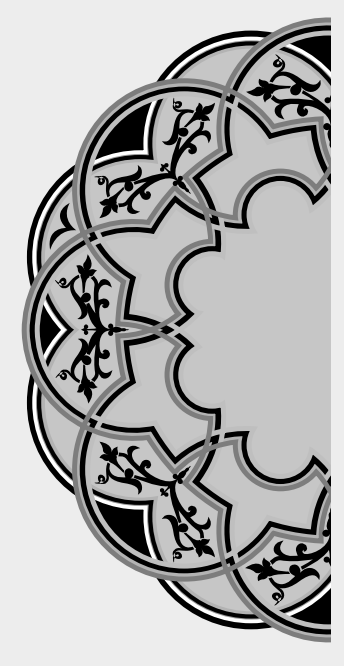

\title{
"If you always do what interests you, at least one person is pleased."
}

\author{
Katharine Hepburn
}

\section{AUTHORSHIP AND CONTRIBUTION DECLARATION}

\begin{tabular}{|c|c|c|c|}
\hline Sr. \# & Author-s Full Name & Contribution to the paper & Author $=\mathbf{s}$ Signature \\
\hline 2 & $\begin{array}{l}\text { Dr. M. Shahbaz Hussain } \\
\text { Dr. Zafar Majeed }\end{array}$ & $\begin{array}{l}\text { Clinical data collection and } \\
\text { analysis, Study design and } \\
\text { Formating and Research concept } \\
\text { Drafting of paper }\end{array}$ & $a^{\prime}$ \\
\hline 3 & Dr. Mazhar Hussain & Study design and formatting & \\
\hline
\end{tabular}

